\title{
Combining Embryology, Anatomy, and Congenital Malformations in Teaching Reproductive Development to Medical Students
}

\author{
Kristen Farraj ${ }^{1}$, Michelle Annabi ${ }^{1}$, Melinda Danowitz ${ }^{1,2}$ and Nikos Solounias ${ }^{1 *}$ \\ ${ }^{1}$ Department of Anatomy, New York Institute of Technology College of Osteopathic Medicine, USA \\ ${ }^{2}$ Department of Pediatrics, A I duPont Hospital for Children, USA
}

*Corresponding author: Nikos Solounias, New York Institute of Technology College of Osteopathic Medicine, USA.

Submission: 想 September 18, 2017; Published: 监 November 13, 2017

\begin{abstract}
The evolutionary history of the male and female reproductive tracts is reflected in the human embryology and adult anatomy. The sexually indifferent gonad consists of primary and secondary sex cords. The primary cords proliferate in the medulla and the secondary cords regress in the male gonad, forming a testis, whereas the secondary sex cords proliferate from the cortex of the female gonad, forming an ovary. The persistence of both primary and secondary sex cords in primitive vertebrates allows for the rare phenomenon of functional hermaphroditism, where the gonad can function as a testis or ovary. The internal genitalia are formed from two sets of ducts; the paramesonephric ducts form the female internal structures and the mesonephric ducts form the male internal structures. The paramesonephric ducts are primitive in many aquatic vertebrates that exhibit external fertilization and development of the embryo, whereas the ducts form a muscular and vascular uterus in mammals, allowing the embryo to develop and grow internally. The male and female external genitalia are formed by the same embryonic precursors, which are feminized in the absence of male hormones, or which undergo virilization in the presence of dihydrotestosterone. The embryology of the male and female reproductive system is reflected in its evolutionary history; therefore a basic knowledge of comparative anatomy facilitates a better understanding of human development.
\end{abstract}

Keywords: Comparative anatomy; Paramesonephric; Mesonephric; Cloaca; Reproductive embryology

\section{Introduction}

Sexual reproduction is the primary mechanism for the production of variation among individuals of different species, in which male and female gametes fuse to form a single zygote. In meiosis, a process of crossing-over, or recombination of genetic material in chromosomes, occurs after fertilization has taken place. This allows for the mixing of genetic material from the male and female, thus creating a gamete with a unique combination of genes. This can lead to the appearance of a new anatomic or physiologic trait. Anatomical structures are adapted to function in various environments by evolution. Features that are well adapted to their surroundings are selected over time, allowing a species to better survive in their ecological niche. Variation of individuals within a species is necessary for any evolutionary process to occur.

The reproductive system is one of few organ systems that is not fully functional at birth. Unlike the heart that starts beating at week 3 of development, or the lungs that begin oxygenating blood immediately as the newborn takes its first breath, the human reproductive system is functionally immature until the individual reaches puberty. The primary function of the reproductive system is the production of gametes and their fertilization and ultimate development into a fetus. In male humans, functional spermatogenesis starts around puberty, and the production of sperm continues throughout the lifetime in the adult. In females however, meiosis for the production of eggs begins around the $5^{\text {th }}$ month in utero. Then, the primary oocytes arrest in prophase of meiosis I, and remain dormant until puberty [1]. Meiosis is completed during fertilization. Human females release eggs monthly until menopause.

The embryologic development and ultimate adult anatomy of the reproductive system reflects the environmental conditions of the individual species. In aquatic vertebrates, the paramesonephric ducts that develop into the female internal genitalia are primitive. In fishes and amphibians that exhibit external fertilization, a specialized uterus is not necessary, as the eggs grow and develop outside the mother. In species that fertilize and develop the embryo internally, such as placental mammals, the duct systems are more specialized and a highly vascular and muscular uterus is present, housing the fetus during gestation.

Comparative evolutionary anatomy is fundamental topic that is rarely taught in the pre-medical or medical curriculum [2]. 
Recent articles have linked evolutionary history to the embryology of head and neck, gastrointestinal system, and respiratory system, to incorporate comparative anatomy into the teaching of medical students [3-5]. A basic understanding of the evolutionary development of the reproductive system puts the human embryology and anatomy into context. We believe a comprehensive review of male and female genital tract development, supplemented with the evolutionary history of each structure, facilitates a deeper understanding of human reproductive embryology and broadens the medical student knowledge base.

\section{Sex development and the indifferent stage}

The sex of an embryo is determined at the moment of fertilization, by the presence of an $\mathrm{X}$ or $\mathrm{Y}$ chromosome on the sperm. The Y chromosome includes the SRY gene that encodes the sex-determining transcription factor (also referred to as testis determining factor, or TDF), which leads to the development of male gonads. In females, due to the lack of Y chromosome and consequent absence of TDF, the gonad differentiates into an ovary. Although the sex of the embryo is determined at conception, up until the $7^{\text {th }}$ week, the development of both the female and male embryos are morphologically sexually indifferent [1].

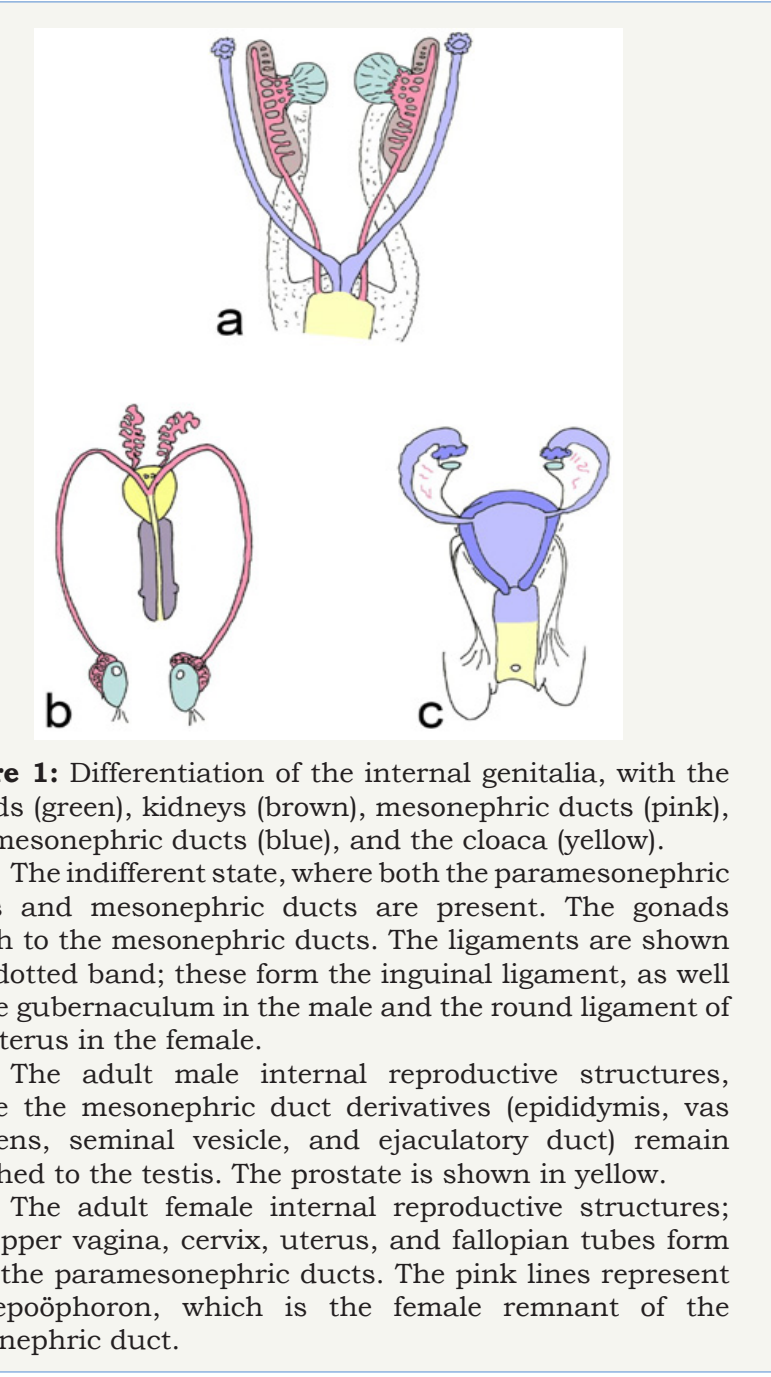

There are two ductal systems that contribute to the formation of internal genitalia (Figure 1). The paramesonephric (Müllerian) ducts form female structures, and the mesonephric (Wolffian) ducts form male structures. Initially, both male and female embryos develop both sets of ducts; the paramesonephric ducts persist in the female and regress in the male, whereas the mesonephric ducts persist in the male and regress in the female [6].

Gonads: Primordial germ cells are the precursors to gametes, and are found in the lining of the yolk sac. Around week 5 of development, these germ cells migrate through the dorsal mesentery of the gut into the intermediate mesoderm, which initiates the formation of the gonads. During the 6th week of development, the germ cells first appear within the genital ridge and the cells begin to differentiate into the gonad [7].

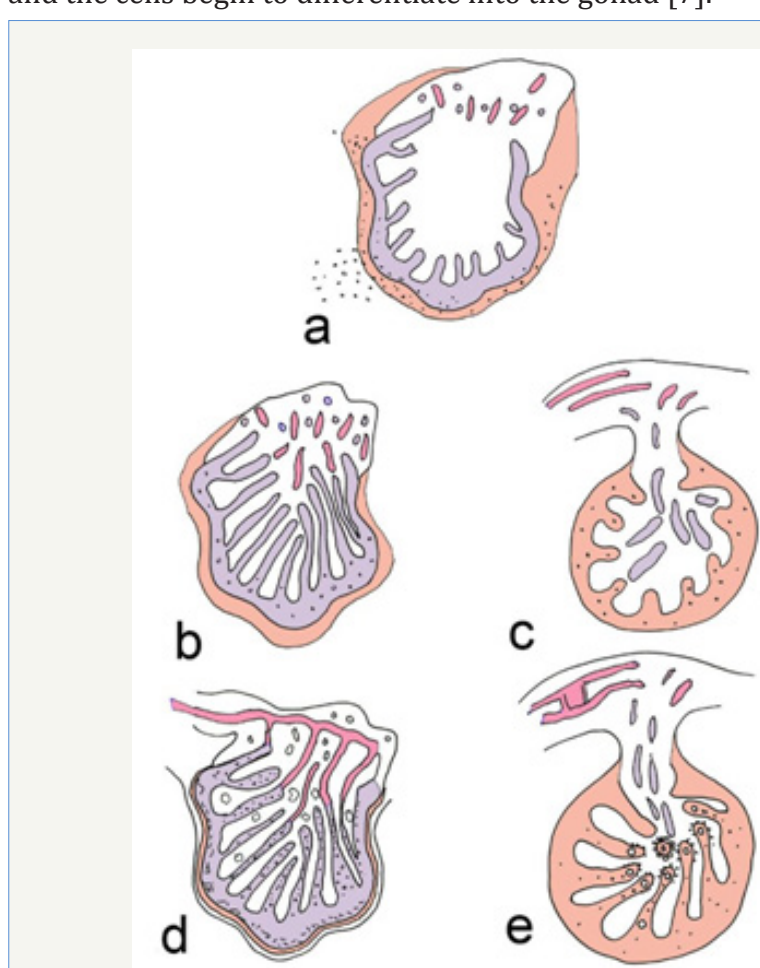

Figure 2: The differentiation of the gonad into a testis or ovary. The primary sex cords are shown in purple, secondary sex cords in peach, and mesonephric duct is represented in pink. The small black dots are primordial germ cells that migrate to the gonads from their original position on the surface of the yolk sac.

a. The indifferent state.

b. Early male testicular formation. The primary sex cords extend from the medulla towards the mesonephric ducts.

c. Early female ovary formation. The secondary sex cords proliferate from the ovarian cortex.

d. Mature testis, where the primary sex cords connect to the mesonephric ducts.

e. Mature ovary, where the secondary sex cords contain sex cells, which will become mature ova in follicles.

The primordial sex cords are present in both sexes, and are necessary to initiate the process of differentiation of the gonad. Without these structures, the ovaries or testes may develop but will ultimately be sterile. In males, the primordial germ cells 
concentrate within the primary sex cords, which extend into the medulla, or inner layer of the gonad to form seminiferous tubules (Figure 2). The primary sex cords are externally surrounded by the tunica albuginea. The primary sex cords connect to the ducts of the mesonephric kidney, which will become the epididymis and vas deferens. In females, the primary sex cords disintegrate along with the tunica albuginea, and secondary sex cords grow from the ovarian surface and extend into the cortex of the ovary. The primordial germ cells in females concentrate in the cortical secondary sex cords and ultimately form follicles. Both the primary and secondary sex cords are derived from endoderm Cochard [8].

Evolution: In most vertebrates that exhibit external fertilization, including various fishes and amphibians, the sperm and eggs contact in the water rather than internally in the female, and the gonads are relatively large compared to their body size, reflecting the large number of gametes released. In species that reproduce on land, such as the reptiles and mammals, the gonads tend to be proportionately smaller; in these species, the internal ducts occupy more space so fertilization and possible internal development of the fetus can occur [9]. Both the primary and secondary sex cords are found in the gonad before the differentiation to the genetically assigned sex. To create a testis, the secondary sex cords disintegrate and in the ovary, the primary sex cords disintegrate. The persistence of both sex cords allows for the development of hermaphroditic individuals. In clownfish, for example, the female is proportionately large due to her primary role in breeding. If the female of a family dies, the male has the ability to change sex to female by competitive overgrowth of the gonadal cortex, therefore ensuring the production of offspring. In most species, however, the gonad maintains its final structure as a testis or ovary throughout the lifecycle of an individual [10].

Amphibians and fishes produce hundreds to thousands of eggs during a single breeding season, but in birds, mammals, and reptiles, much fewer mature eggs are produced at once. As vertebrates invade the land, the number of eggs released in a mating season dramatically decreases, which allows the individual follicles to enlarge. In placental mammals, where both fertilization and fetal development occur internally, the egg is small but the follicle surrounding it is large. The yolk becomes consumed early in gestation; however the mother provides a constant supply of nutrients to the fetus via the placenta [11].

\section{Development of the paramesonephric ducts}

The female embryo initially possesses both paramesonephric and mesonephric ducts. The paramesonephric ducts develop lateral to the mesonephric ducts. The two paramesonephric ducts fuse distally at the midline; this fusion forms the uterus, cervix and upper $1 / 3$ of the vagina. The proximal unfused ducts develop into the fallopian tubes. Unlike in male development, where testosterone is necessary for maturation of the mesonephric duct, there are no hormones necessary for the differentiation of female internal genitalia; the maintenance of the paramesonephric ducts and distal fusion occur by default. Since the mesonephric ducts require testosterone to maintain the cells, the lack of testosterone production in the female leads to the disintegration of the mesonephric ducts. The mesonephric ducts, however, form small remnants in the adult female: the epoöphoron and paroöphoron, which are found in the mesovarium, or the mesentery of the ovary [6].

Evolution: As vertebrates become land-adapted, the paramesonephric duct derivatives become more specialized, allowing for gamete transport within the female individual. The evolution of the oviduct allows for a more specialized mode of egg transport. In certain fishes that release massive quantities of eggs during breeding, the ovary connects directly with the oviduct, therefore preventing the released gametes from filling the celom. In most species, such as in humans, the ovary is separate from the oviduct and the ciliated fallopian tube functions in catching the eggs and directing them towards the uterus [12].

In some species where the embryo develops externally, including sharks, rays, reptiles and birds, an eggshell serves a protective external covering for the growing embryo. The oviduct of these groups forms specialized regions for producing egg white and a shell gland. It is rich in calcium, and the calcium reservoir is used to form the skeleton of the developing embryo, as there is no persistent maternal blood supply providing the necessary minerals [10].

Amniotes is a general term that includes mammals, birds, and reptiles, which is defined by the presence of two membranes that surround the embryo, the amnion and the chorion. Mammalian oviducts do not produce the shell or the albumin secreting glands, because the growing embryo will be housed in the uterus. The uterus of placental mammals and marsupials is the site of the development of the embryo, and therefore the uterine endometrium is rich in vasculature. This increase in blood supply allows for the transport of nutrients from the mother, and the excretion of wastes from the fetus. The uterine wall is thick and muscular, which supports the fetus during gestation and allows for contractions during delivery. The thickness of the uterine wall is dependent on ovarian hormones and molecular signals from the fetus [12].

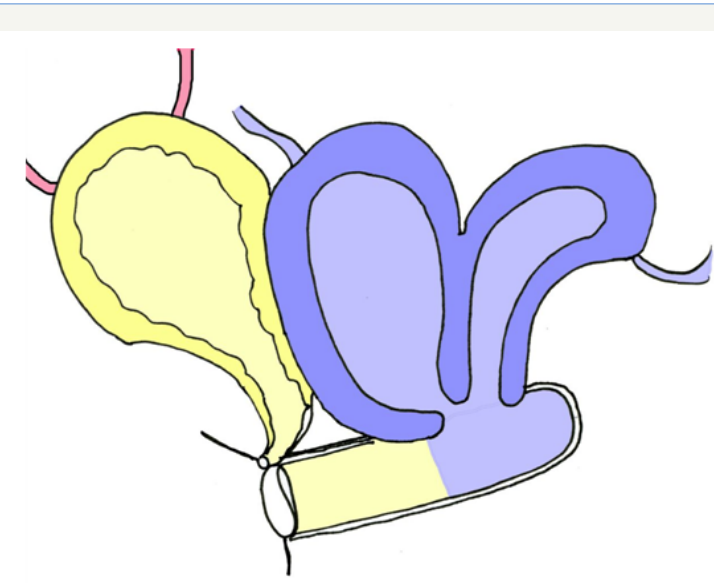

Figure 3: Schematic of a bicornuate uterus. In this condition the paramesonephric ducts do not fuse completely. This represents a congenital anomaly in humans, and a normal variant in certain mammals. Cloacal derivatives are shown in yellow and paramesonephric derivatives are shown in blue. 
In primitive mammals, the paramesonephric ducts remain unfused, therefore forming two separate uteri. In more specialized species, the distal paramesonephric ducts fuse, forming a bicornuate uterus (Figure 3). Interestingly, this resembles a congenital anomaly that is sometimes seen in humans. In most primates, including humans, a complete fusion forms a single midline uterus [11].

\section{Development of the mesonephric ducts}

The differentiation of an embryo into a male is initiated by the presence of the SRY gene on the Y chromosome. This gene allows for the differentiation of somatic support cells into Sertoli cells, which initiate the formation of the seminiferous tubules. Sertoli cells also secrete the glycoprotein Anti-Müllerian hormone (AMH; also referred to as Müllerian inhibiting factor, or MIF), which causes the disintegration of the paramesonephric ducts. Without Anti-Müllerian hormone or receptors, the paramesonephric ducts would persist and form female internal genitalia, as the cells form by default. The male adult remnant of the paramesonephric ducts is the appendix testis and prostatic urethra. Leydig cells differentiate in the testes around weeks 9-10 of development, and are responsible for the secretion of testosterone. Testosterone binds to the cells of the mesonephric duct, causing the differentiation of the ducts into the male internal genitalia, including the seminal vesicles, epididymis, and vas deferens. The cells of the mesonephric duct are maintained and matured only in the presence of sufficient testosterone by the Leydig cells (Figure 4). The rete testis forms the connection between the mesonephric duct and the seminiferous tubules within the testes. This pathway canalizes and becomes patent during puberty. In both sexes, the mesonephric duct also forms the ureteric bud, which develops into distal kidney structures, and forms the trigone of the bladder [1].

\begin{tabular}{|c|c|c|c|c|}
\hline Ovary & $\begin{array}{c}\text { Primordial germ } \\
\text { cells }\end{array}$ & Indifferent gonad & $\begin{array}{c}\text { TDF from SAY } \\
\text { gene }\end{array}$ & Testis \\
\hline $\begin{array}{l}\text { Gartner's duct } \\
\text { Epoöphoron } \\
\text { Paroöphoron }\end{array}$ & & $\begin{array}{l}\text { Mesonephric } \\
\text { duct/tubules }\end{array}$ & Testosterone & $\begin{array}{l}\text { Seminal vesicle } \\
\text { Ejaculatory duct } \\
\text { Epididymis } \\
\text { Vas deferens }\end{array}$ \\
\hline $\begin{array}{c}\text { Fallopian tubes } \\
\text { Uterus } \\
\text { Cervix } \\
\text { Upper } 1 / 3 \text { of vagina }\end{array}$ & & $\begin{array}{l}\text { Paramesonephric } \\
\text { duct }\end{array}$ & MIF & Appendix of testis \\
\hline $\begin{array}{c}\text { Urethra } \\
\text { Lower } 2 / 3 \text { vagina }\end{array}$ & & Urogenital sinus & DHT & $\begin{array}{l}\text { Urethra (membranous } \\
\text { and prostatic) } \\
\text { Prostate } \\
\text { Bulbourethral glands }\end{array}$ \\
\hline $\begin{array}{l}\text { Glans clitoris } \\
\text { Corpus cavernosa }\end{array}$ & & Genital tubercle & DHT & $\begin{array}{l}\text { Glans penis } \\
\text { Corpus cavernosa } \\
\text { Corpus spongiosum }\end{array}$ \\
\hline Labia minora & & Urogenital folds & DHT & Ventral penis \\
\hline Labia majora & 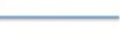 & Labioscrotal folds & DHT & Scrotum \\
\hline \multicolumn{5}{|c|}{$\begin{array}{l}\text { Figure 4: Summary of the embryonic precursors (middle } \\
\text { column) and their adult derivatives. Female structures are } \\
\text { listed on the left with a blue line, and male structures are } \\
\text { listed on the right with a red line. Hormones or molecules } \\
\text { necessary for the development of the precursor into its adult } \\
\text { structure are shown. Notice each female structure forms } \\
\text { without hormonal involvement, whereas male structures } \\
\text { require additional hormones. }\end{array}$} \\
\hline
\end{tabular}

Evolution: The mesonephric duct has a dual role in the development of the male and female excretory systems, as well as in the formation of male internal genitalia. In the primitive cyclostomes, the mesonephric duct does not contact the testes, and the testes release the sperm externally through abdominal pores. Their duct system lacks a reproductive role, and instead exclusively drains the kidneys. In most other groups, the male reproductive system "hijacks" the draining system of the mesonephric kidneys, hence the scientific term "urogenital system." The degree of shared ductal drainage between the urinary and genital systems varies throughout evolution. In certain groups, such as sharks and amphibians, the testes and the urinary ducts directly drain into the mesonephric ducts (Figure 5). In amniotes, the vas deferens and epididymis form from the mesonephric duct, which exclusively drains sperm. Amniotes develop a specialized metanephric kidney as a diverticulum from the distal mesonephric duct, therefore the excretory and genital systems have a common distal drainage [11].

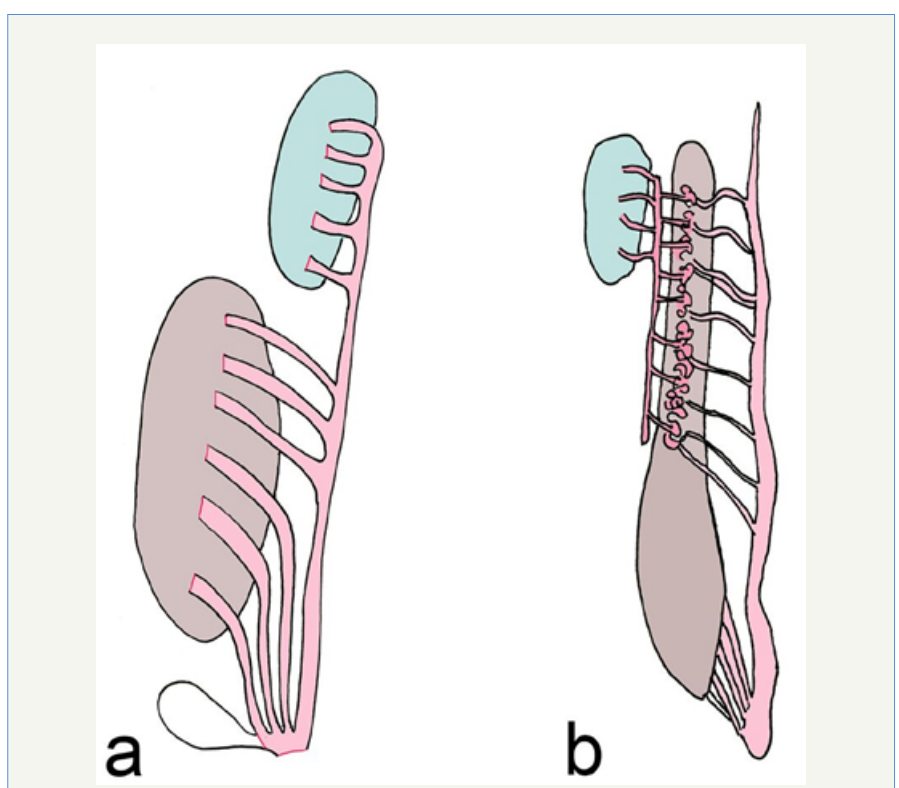

Figure 5: The urogenital system of two non-amniotes. Testes are shown in green, mesonephric ducts in pink, and kidney in brown.

a. The gonadal drainage of a shark (Chondrichthyes). Both the kidney and the testis drains into the mesonephric duct, and both systems exit via a common opening. There is a small diverticulum distally termed the sperm sac.

b. The urogenital drainage of a salamander (Amphibia). The testes indirectly connects to the mesonephric duct via retes in the kidney. Both the kidney and testes ultimately drain into the mesonephric duct.

\section{Cloaca}

Initially, the distal gastrointestinal, urinary, and reproductive tracts have a common opening termed the cloaca. Later in development, the cloaca subdivides anteriorly into the urogenital sinus and posteriorly into the anorectal canal. This division is formed by the urorectal septum, which separates the urogenital system from the gastrointestinal tract (Figure 6). The urogenital sinus forms the bladder and urethra of both sexes, as well as the prostate of males and vestibule of the vagina in females. The formation of the prostate is dependent on the production of dihydrotestosterone [7]. 

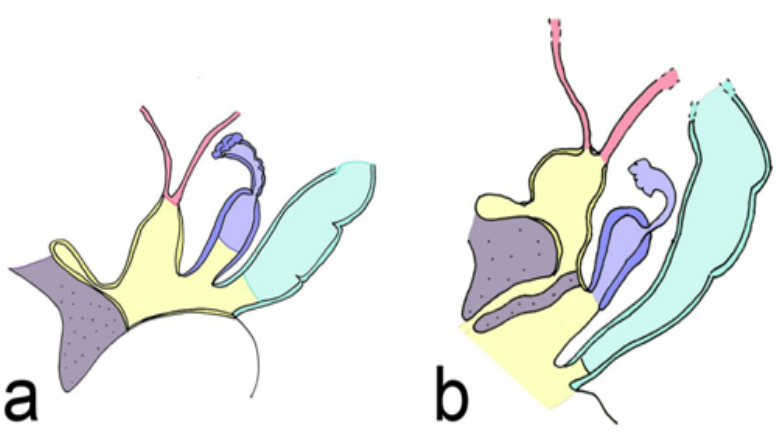

Figure 6: The cloaca and its derivatives in a female. The cloaca is shown in yellow, genital tubercle in purple, paramesonephric duct in blue, and endoderm of the gastrointestinal tract in turquoise. a Early stage, where the distal urinary, reproductive, and gastrointestinal tracts all have a common opening. The anterior out pouching towards the genital tubercle represents the allantois, which is an early drainage system for the fetus. b Later stage where the urogenital system is separated from the distal GI tract via the urorectal septum. From anterior (left) to posterior (right), structures include the bladder and urethra, developing uterus and vagina, and anorectal canal.

Evolution: In all vertebrates, the external genitalia are partially formed from the cloaca, which is a cavity that contains the distal aspect of the reproductive, urinary, and gastrointestinal tracts. All species possess a cloaca at some early point during development, and in numerous species, it persists into adulthood as a common distal opening of all three systems. In the species where the cloaca is maintained, this structure contains separate compartments or regions that function in defecation, urination, or copulation. The cloaca remains as the adult reproductive structure of most reptiles and birds [11,13].

\section{Descent of the Testes}

Initially, the testes and ovaries are positioned high in the abdominal cavity. The ovaries descend a short distance, whereas the testes descend completely into the pelvis and eventually into the scrotal sac. The testes are pulled caudally by the ligamentous gubernaculum, which progressively shortens and ultimately anchors them to their final position in the scrotum. The testes travel through the deep and superficial inguinal rings and create a temporary opening from the abdominal cavity into the pelvis, termed the processusvaginalis. If the processusvaginalis remains patent, this serves as a potential portal for an indirect hernia [14].

Evolution: The descent of the testes into the pelvis is a phenomenon largely unique to mammals; in most other vertebrates, the testes retain their original position in the abdomen. Although the evolutionary drive for testicular descent is debated, many hypothesize that the change in position was for temperature regulation. The positioning of the testes external to the body cavity in the scrotum provides a cooler temperature, which may optimize sperm production and overall fertility. In mammals, the body temperature is maintained at a higher level, which may not be conducive to maximal fertility and testicular function. Certain rodents cyclically ascend and descend their testes via the cremaster muscle, allowing the position to change based on the mating season, environmental conditions, or temperature. In mammals, the pampiniform plexus of veins surrounds the spermatic cord and further regulates the temperature of the sperm. In birds, where the testes are positioned internally, air sacs surround the gonads, therefore cooling the sperm.

Another theory on the driving force for testicular descent is to protect gonadal function during galloping. Galloping involves great fluctuations in intra-abdominal pressure, which can alter blood flow to coelomic gonads, and impede overall function. In addition, as the male reproductive tract lacks sphincters, increased peritoneal pressure during galloping could lead to the release of sperm outside of copulation. Correspondingly, many groups that gallop, including ruminants and horses, have testicles positioned external to the abdominal cavity in scrotal sacs. Most aquatic mammals, including whales and dolphins, have undescended, or internal testes, possibly relating to streamlined body structure to optimize swimming. The elephants evolved from a line of aquatic mammals, and although the massive species is now adapted to live on land, it still retains the intra-abdominal testicular position, reflecting its 50 million year old ancestors (Figure 7) [15].

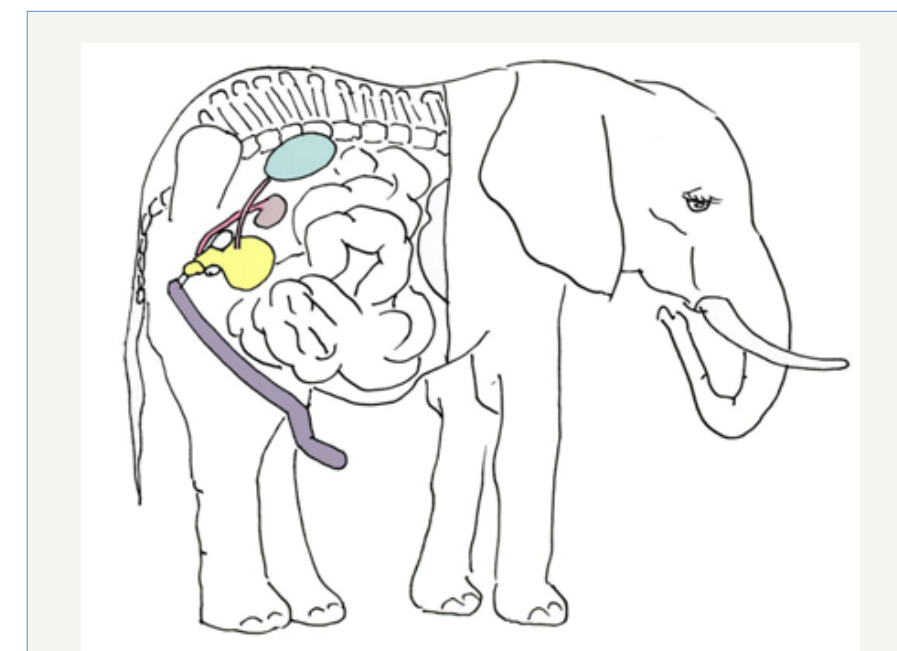

Figure 7: Schematic of a male Indian elephant showing that the testis is undescended and positioned high in the abdomen. The remainder of the reproductive system is the normal location to that of all mammals.

\section{Formation of male external genitalia}

The maturation of the mesonephric duct into male internal structures requires testosterone. The virilization of the external genitalia requires a testosterone derivative, dihydrotestosterone (DHT). Testosterone secreted from the Leydig cells is converted to DHT via the enzyme 5 alpha-reductase. The male and female external genitalia form from the same common precursors: the genital tubercle, urogenital folds, and labioscrotal swellings. The masculinization or feminization of these structures is determined by the presence of, or absence of, DHT [14].

The genital tubercle is a mesodermal swelling positioned anterior to the urogenital sinus. Under the effects of DHT, the genital tubercle lengthens to form the phallus of the penis. The urogenital 
folds form as paired mesodermal swellings. The urogenital folds grow towards each other in the midline and eventually fuse as the phallus lengthens, forming the ventral surface of the penis and penile urethra. The urethra is therefore enclosed in the phallus, within the corpus spongiosum. The labioscrotal swellings are positioned lateral to the urogenital folds; these fuse centrally to form the scrotum (Figure 8). The midline fusion of the urogenital folds and labioscrotal swellings, as well as the elongation of the genital tubercle into the phallus occurs due to the presence of sufficient DHT; without this hormone, masculinization of these structures does not occur. The effects of DHT on the male genitalia become most apparent around the fourth month of development [1].

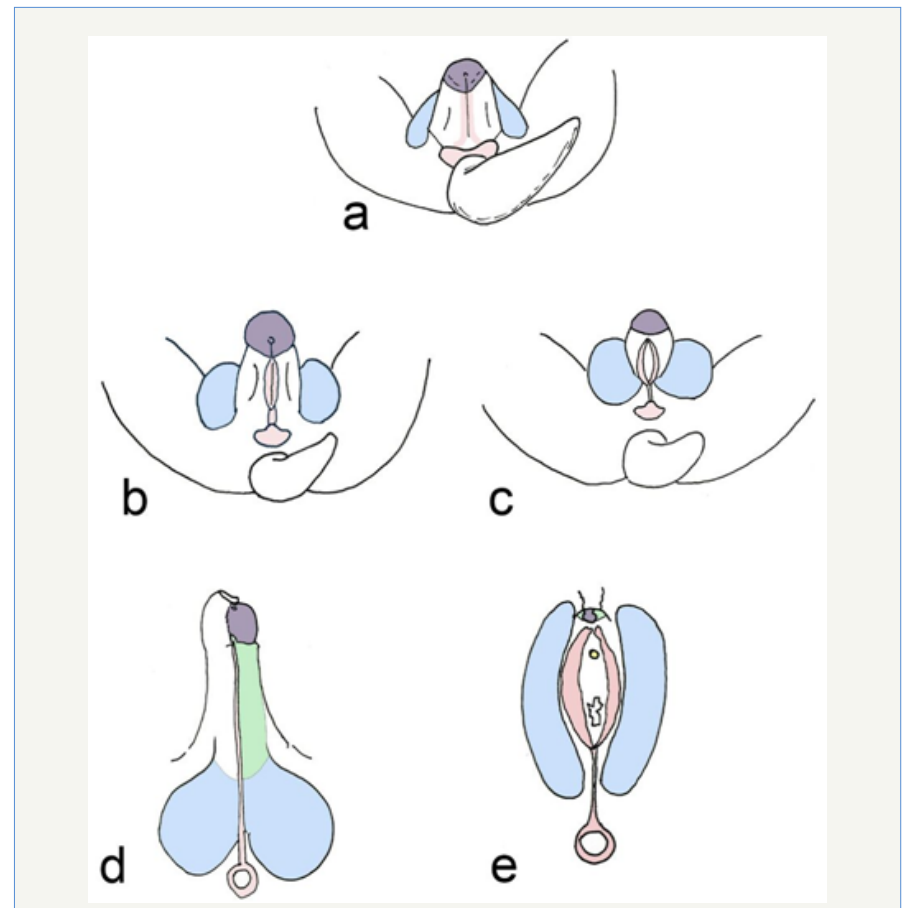

Figure 8: Differentiation of the external genitalia. Genital tubercle is shown in purple, urogenital folds in peach, labioscrotal swellings in blue, and lateral tubercle in green. a. The indifferent state. The urogenital folds connect to the anal tubercle. At this stage, the tail is large.

b. Early male genitalia differentiation. Under the influence of dihydrotestosterone (DHT), the genital tubercle elongates, and the labioscrotal swellings and urogenital folds grow towards the midline.

c. Early female genitalia differentiation.

d. Male external genitalia. The phallus elongates and forms a shaft, the scrotal skin becomes rugated and fuses at the midline, and the urogenital folds fuse at the midline to form the ventral penis and penile urethra, all due to the effects of DHT. Foreskin is shown on the left.

e. Female external genitalia. Without the effects of DHT, the phallus does not elongate and the genital tubercle forms the clitoral head, and the labioscrotal swellings and urogenital folds remain separate, lateral structures.

\section{Formation of female external genitalia}

In the female, the genital tubercle differentiates into the glans clitoris and corpus cavernosum. The urogenital folds remain separate and develop into the labia minora. Lateral to the urogenital folds is a pair of labioscrotal swellings, which form the labia majora (Figure 8). The urethra develops inferior to the clitoral phallus. Both male and female embryos possess the genital tubercle, urogenital folds, and labioscrotalswellings; the differentiation of these structures into female external genitalia is biologically the default state. In other words, the feminization of the external genitalia does not require estrogen, or any additional hormones [6].

The urogenital sinus from the cloaca develops into the lower $2 / 3$ of the vagina, membranous urethra, glands of Bartholin and Skene, and the bladder. The pelvic portion of the urogenital sinus condenses into endodermal swellings called the sinuvaginal bulbs; these bulbs fuse to form the vaginal plate. This plate subsequently is canalized, connecting the vaginal vestibule with the lower portion of the vagina. The adult hymen is the remnant of this membranous separation between the vaginal lumen and vestibule [8].

Evolution: Species that exhibit internal fertilization require genitalia that allow for the passage of male sperm into the female internal structures. In sharks, claspers develop as extensions from the pelvic fin. The claspers are responsible for hooking into the cloaca of the female to ensure sperm transport. Certain reptiles exhibit penile extensions that insert into the female during copulation. The mammalian penis is an adaptation that works as a hydrovascular mechanism, where the circulatory system along with the parasympathetic system can control the stiffness of the penis. During mating, the spongy tissues of the mammalian penis, the corpora cavernosa and corpus spongiosum fill with blood, causing a stiffening of this organ, allowing it to penetrate into the female. The erection is maintained by a tough coat, the tunica albuginea, which surrounds the penis. Some mammalian species exhibit an additional bony structure termed the baculum, which aids in maintaining stiffness during mating; the baculum is located in between the two corpora cavernosa (Figure 9) [11].

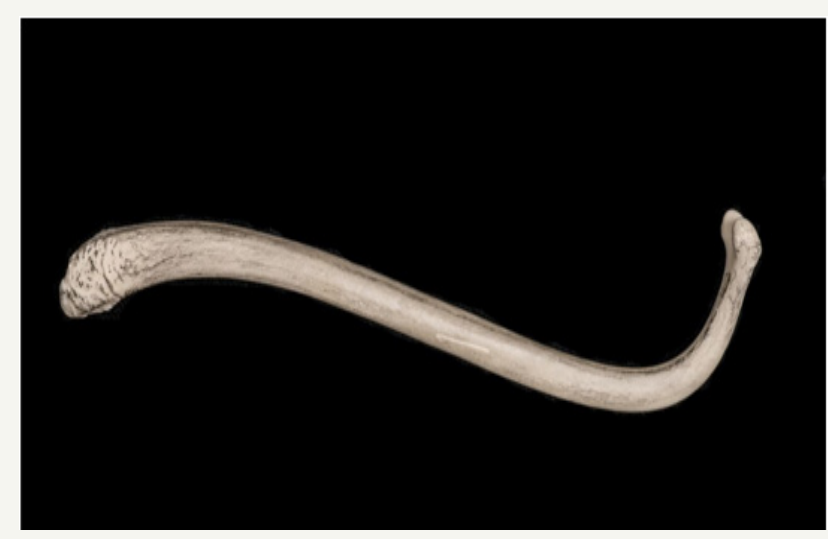

Figure 9: The baculum (os penis) of a North American raccoon (Procyon). This symmetrical bone is situated between the two corpora cavernosa of the penis. It is found in many mammals and allows for the maintenance of a stiffened penis during mating.

\section{Female congenital abnormalities}

Müllerian agenesis (Mayer-Rokitansky-Küster-Hauser syndrome): Regression of the paramesonephric ducts in a 
female, resulting in a lack of uterus and fallopian tubes. This presents with a shortened, blind-ending vaginal canal and primary amenorrhea. Ovaries are present and functional, and therefore estrogen production and development of female secondary sex characteristics is normal [16].

\section{Congenital adrenal hyperplasia}

Deficiency of an adrenal enzyme, leading to dysfunctional adrenal hormone, and steroid production. The most common variant is 21-hydroxylase deficiency, preventing production of glucocorticoids and mineralocorticoids. The lack of aldosterone and glucocorticoids leads to hypotension due to volume depletion, hyperkalemia, and hypoglycemia. 17-hydroxyprogesterone, the precursor of the deficient enzyme, is shunted to the sex hormone pathway, leading to increased adrenal production of androgens. The excess androgens are converted peripherally to testosterone and DHT, leading to virilization of female external genitalia and retention of the mesonephric ducts [17].

\section{Imperforate hymen}

Failure of the sinuvaginal bulbs to canalize, leading to a blockage in the vaginal canal. This is often diagnosed at puberty as menstrual flow is blocked, leading to hematocolpos formation and cyclical pelvic pain [18].

\section{Male congenital abnormalities}

Congenital adrenal hyperplasia: Deficiency of an adrenal enzyme, leading to dysfunctional adrenal hormone, and steroid production, two less common variants include 3 beta hydroxysteroid deficiency, and 17 alpha hydroxylase deficiency, both of which lead to a blockage in the adrenal and testicular sex hormone synthesis pathways. Decreased production of testosterone and DHT leads to undervirilization of the internal and external genitalia, resulting in partial or complete regression of the mesonephric duct and ambiguous or feminized external genitalia [17].

Persistent Müllerian duct syndrome: Mutation of absence of the AMH receptors, preventing the regression of the paramesonephric ducts. This would present as a genotypic and phenotypic male with normal male internal and external genitalia, as testosterone production is unaffected, but with the anomalous presence of a uterus, cervix, and fallopian tubes (Figure 10) [19].

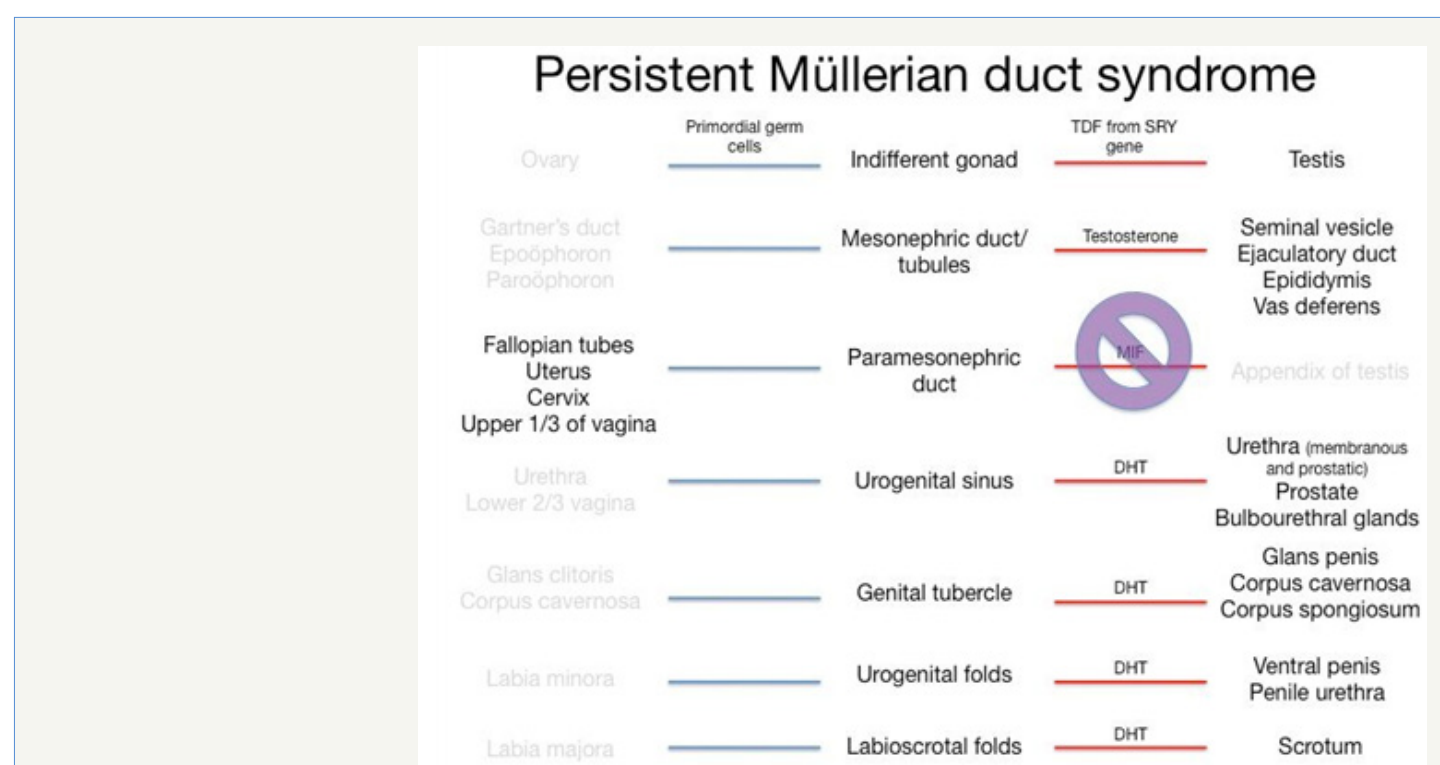

Figure 10: Persistent Müllerian duct syndrome. A male with this pathology has mutated or absent receptors to Müllerian inhibiting factor (MIF, also known as anti-Müllerian hormone), therefore preventing the regression of the paramesonephric ducts. The ducts are therefore maintained, and will form a uterus with fallopian tubes in an otherwise normal male individual.

Epispadias: Improper positioning of the genital tubercle, leading to an anomalous urethral opening, on the dorsal surface of the penis. This can be associated with exstrophy of the bladder [20].

Hypospadias: Failure of complete fusion of the urethral folds, leading to an anomalous urethral opening on the ventral, or undersurface of the penis [21].

Cryptorchidism: Failure of one or both testes to descend. The testis will often descend spontaneously, or can be brought down surgically via orchiopexy if undescended after 1 year of life. The incidence is increased in disorders such as androgen insensitivity syndrome, Kallman syndrome, and persistent Müllerian duct syndrome. This leads to suboptimal fertility and increased risk for malignancy, however testosterone production is unaffected by the anomalous testicular positioning [22].

\section{Androgen insensitivity syndrome (testicular feminization syndrome)}

Mutated or absent receptors for both testosterone and DHT. Although male steroid hormone synthesis is normal, the target tissues lack functional androgen receptors. This prevents testosterone from binding to the mesonephric ducts, which will therefore regress, preventing the formation of male internal genitalia. This also prevents the effects of DHT on the genital tubercle 
and urogenital folds, leading to ambiguous or feminine external genitalia. The production of and receptors for AMH is unaffected, therefore the paramesonephric ducts regress, and no female internal genitalia are present. At birth, males are often mistaken for female due to the feminized external genitalia, and diagnosis is made at puberty when the adolescent never menstruates (Figure 11) [23].

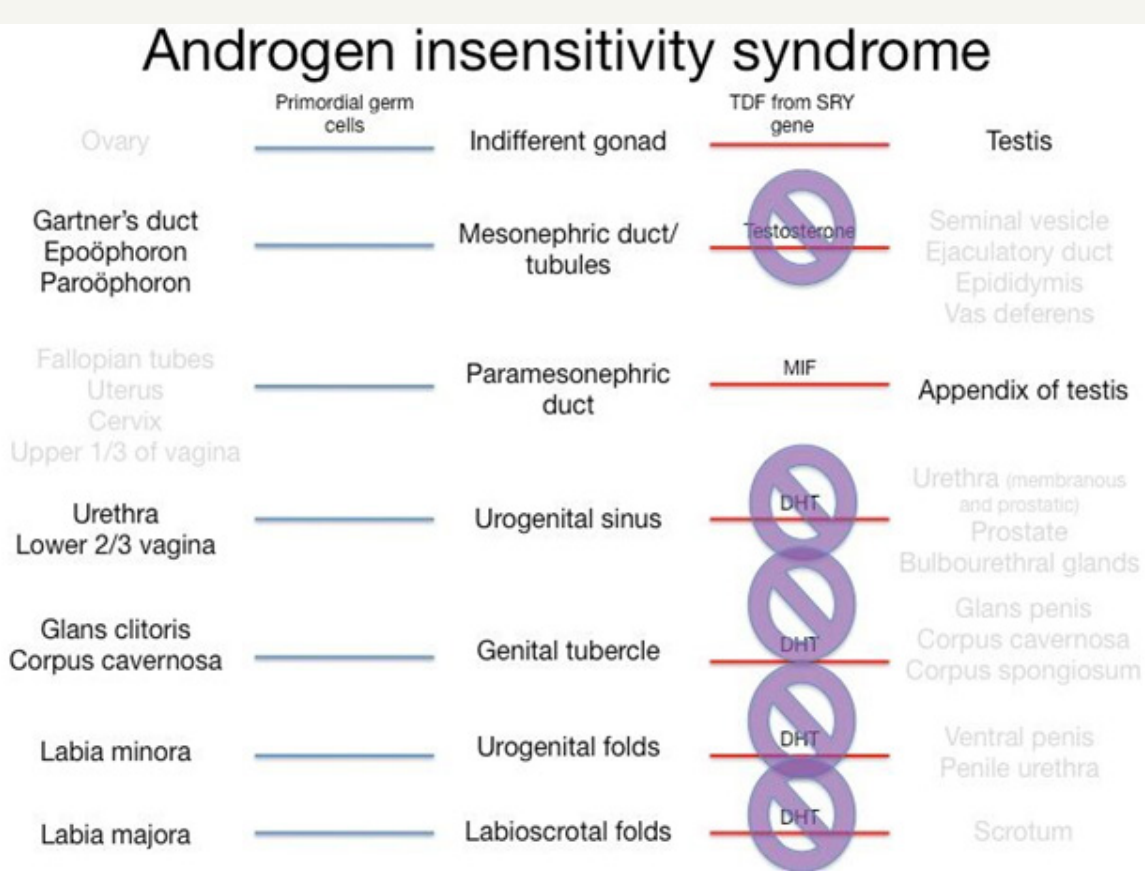

Figure 11: Androgen insensitivity syndrome. In this pathology, the receptors to male androgens (testosterone and DHT) are mutated or absent, therefore the virilization of the internal and external genitalia is prevented. In complete androgen insensitivity syndrome, the individual has female appearing external genitalia, and lacks internal genitalia of either sex.

\section{Alpha reductase defiiency}

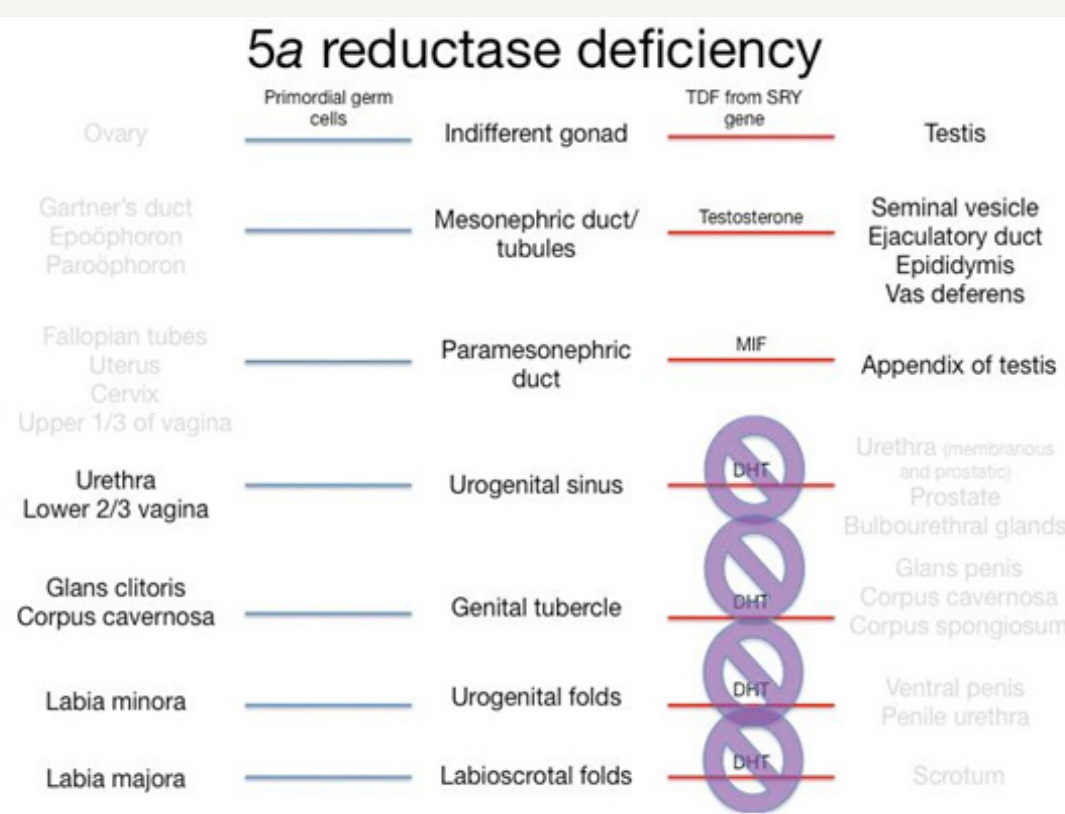

Figure 12: 5 alpha reductase deficiency. In this pathology, the enzyme that converts testosterone to DHT is non-functioning, causing ineffective development of male external genitalia. Internal male development is normal, as there is sufficient testosterone production to maintain the mesonephric ducts. 
The enzyme that converts testosterone to DHT (5 alpha reductase) does not work properly, however testosterone production is normal. Since there is abundant testosterone, development of the mesonephric duct into internal male structures is unaffected. However, there is ineffective development of the external male genitalia and prostate due to lack of sufficient DHT. As in androgen insensitivity syndrome, these males are misdiagnosed as female at birth due to feminized external genitalia, but they never commence menstruation as they lack ovaries and a uterus. During puberty, the testosterone surge often overcomes the enzyme deficiency, and allows for sufficient DHT production to cause some virilization (Figure 12) [24].

\section{Male and female congenital abnormalities}

Imperforate anus: Dysfunctional positioning, or absence of the urorectal septum, which normally separates the cloaca into the urogenital sinus and anorectal canal. Several variants exist, depending on the location of the anomalous septum, including the rectum opening into the reproductive tract, urinary tract, or the rectum ending in a blind pouch. This presents as failure to pass meconium due to an anomalous distal gastrointestinal tract, and the presence of a dimple in the place of the anus. This is part of the "VACTERL" association, which includes vertebral anomalies, anal atresia, cardiovascular anomalies, tracheoesophageal fistula, esophageal atresia, renal anomalies, and limb defects [25].

\section{Methodology}

The above review was written using well-known embryology and comparative evolutionary anatomy textbooks as well as relevant journal articles. The information on human embryology was summarized and aspects most pertinent to the medical student education were emphasized. The comparative anatomy and evolution sections were written to include aspects of human evolution and interesting examples of comparative anatomy.

\section{Discussion}

Combining human embryology and comparative evolutionary anatomy broadens the education of medical students and puts development into perspective. Unfortunately, comparative anatomy is rarely included in the medical and pre-medical education; however, a general understanding is conducive to a deeper understanding of human development and anatomy. The purpose of this review article is to help medical students understand why humans develop the way we do, rather than simply memorizing why, by showing examples of how structure relates to function and how anatomical structures have evolved to fit a specific purpose.

\section{Conclusion}

The differentiation of female internal and external genitalia is not dependent on hormonal influence. The paramesonephric duct forms the fallopian tubes, uterus, and upper portion of the vagina, the genital tubercle forms the clitoris, and the urogenital folds and labioscrotal swellings form the labia minora and majora, respectively. Due to a lack of testosterone, the mesonephric ducts regress, by default, in the female. Male development, however, is highly dependent on hormones such as testosterone, and its derivative dihydrotestosterone.

Male testis secretes Anti-Müllerianhormone, which leads to the disintegration of the paramesonephric ducts, and testosterone, which causes the maintenance of the mesonephric ducts and maturation into the male internal genitalia. The external precursors are virillized under the influence of dihydrotestosterone, causing the elongation of the phallus, and growth and midline fusion of the scrotum. Dihydrotestosterone is also responsible for the development of the prostate from the urogenital sinus. The embryology of the male and female reproductive system is reflected in its evolutionary history; therefore a basic knowledge of comparative anatomy facilitates a better understanding of human development.

\section{References}

1. Schoenwolf GC, Bleyl SB, Braue PR, Francis-West PH (2009) Larsen's human embryology ( $4^{\text {th }}$ edn), Churchill Livingstone, USA.

2. Nesse RM, Schiffman JD (2003) Evolutionary Biology in the Medical School Curriculum. Bio Science 53(6): 585-587.

3. Danowitz M, Zheng H, Guigova A, Solounias N (2016) A combined approach of teaching head development using embryology and comparative anatomy. J Anat Embryo 3: 17-27.

4. Danowitz M, Solounias N (2016) Embryology, comparative anatomy, and congenital malformations of the gastrointestinal tract. J Anat Embryo 3: 39-50.

5. White S, Danowitz M, Solounias N (2016) Embryology and evolutionary history of the respiratory tract. J Anat Embryo 3: 54-62.

6. Sadler TW (2014) Langman's Medical Embryology (13 ${ }^{\text {th }}$ edn), Lippincott Williams \& Wilkins, USA.

7. Hamilton WJ, Mossman HW (1972) Human embryology (4 $4^{\text {th }}$ edn), Williams and Wilkins Company, USA.

8. Cochard LR (2012) Netter's atlas of human embryology. Saunders, USA, p. 288.

9. Wake MH (1979) Hyman's Comparative Vertebrate Anatomy ( $3^{\text {rd }}$ edn), University of Chicago Press, USA.

10. Kardong KV (2014) Vertebrates: comparative anatomy, function, evolution ( $7^{\text {th }}$ edn), McGraw-Hill, USA.

11. Romer AS, Parsons TS (1986) The Vertebrate Body ( $5^{\text {th }}$ edn), WB Saunders Company, USA.

12. Feduccia A, McCrady E (1991) Torrey's Morphogenesis of the Vertebrates $\left(5^{\text {th }}\right.$ edn), John Wiley \& Sons, USA.

13. Brennan PLR, Prum RO, McCracken KG, Sorenson MD, Wilson RE, et al. (2007) Coevolution of male and female genital morphology in waterfowl. PLoS ONE 2(5): e418.

14. Standring S (2015) Gray's anatomy: the anatomical basis of clinical practice ( $41^{\text {st }}$ edn), Churchill Livingstone, USA.

15. Kleisner K, Ivell R, Flegr J (2010) The evolutionary history of testicular externalization and the origin of the scrotum. J Biosci 35(1): 27-37.

16. Folch M, Pigem I, Konje JC (2000) Müllerian agenesis: etiology, diagnosis, and management. Obstet Gynecol Surv 55: 644-649.

17. Speiser PW, White PC (2003) Congenital adrenal hyperplasia. NEJM 349: 776-788.

18. Dane C, Dane B, Erginbas M, Cetin A (2007) Imperforate hymen- a rare cause of abdominal pain: two cases and review of the literature.J Pediatr Adolesc Gynecol 20(4): 245-247. 
19. Buchholz NP, Biyabani R, Herzig MJ, Al A, Nazi Z, et al. (1998) Persistent Müllerian duct syndrome. Eur Urol 34: 230-232.

20. Woodhouse CR, Kellett MJ (1984) Anatomy of the penis and its deformities in extrophy and epispadias. J Urol 132(6): 1122-1124.

21. Baskin LS, Ebbers MB (2006) Hypospadias: anatomy, etiology, and technique. J Pediatr Surg 41(3): 463-472.

22. Wensing CJG (1988) The embryology of testicular descent. Horm Res Paediatr 30: 144-152.
23. Hughes IA, Davies JD, Bunch TI, Pasterski V, Mastroyannopoulou K, et al. (2012) Androgen insensitivity syndrome. Lancet 380 (9851): 14191428.

24. Sultan C, Paris F, Terouanne B, Balaguer P, Georget V, et al. (2001) Disorders linked to insufficient androgen action in male children. Hum Reprod Update 7(3): 314-322.

25. Stafford SJ, Klein MD (2011) Surgical conditions of the anus and rectum. In: Kliegman RM, Behrman RE, Jenson HB, Stanton BF (Eds.), (19 ${ }^{\text {th }}$ edn), Nelson Textbook of Pediatrics, Elsevier Saunders, USA. 